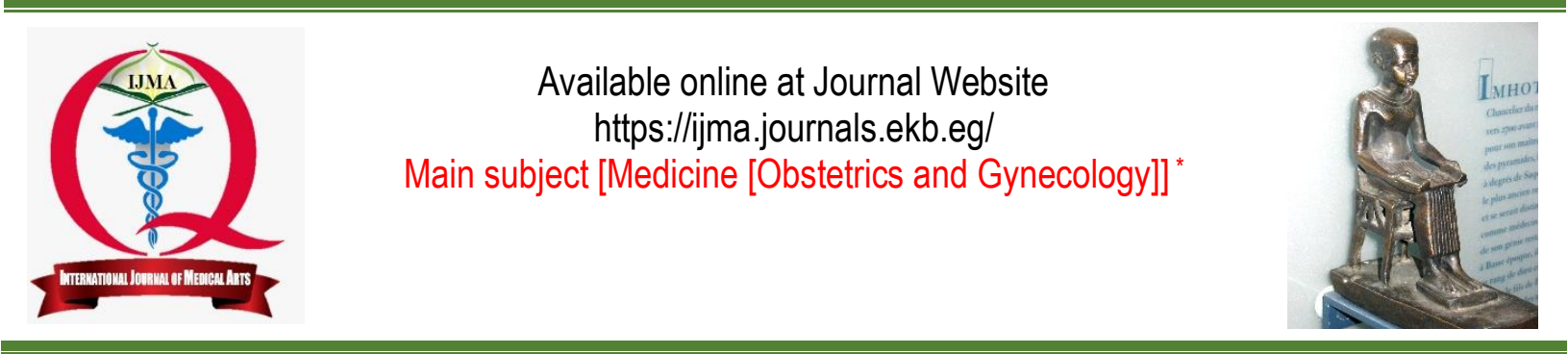

Original article

\title{
Maternal Plasma Lipid Concentration in Third Trimester of Women with Preeclampsia and Normotensive Pregnancy
}

\author{
Ghada Khalil Mohammed ${ }^{\mathbf{a}}$; Waleed Ahmed Ayad ${ }^{\mathbf{b}}$; Usama Bhgat El-Gazzar $\mathbf{c}$; Samia Mohmad Eid ${ }^{\mathbf{b}}$ \\ Department of Obstetrics and Gynecology, Nabaruh General Hospital, Ministry of Health, Egypt [a]. \\ Department of Obstetrics and Gynecology, Damietta Faculty of Medicine, Al-Azhar University, Egypt ${ }^{[\mathrm{b}]}$. \\ Department of Medical Biochemistry, Damietta Faculty of Medicine, Al-Azhar University, Egypt ${ }^{[b]}$.
}

Corresponding author: Ghada Khalil Mohammed

Email: ghadakhalil2018@gmail.com

Received at: February 10, 2020; Revised at: March 20, 2020; Accepted at: March 20, 2020; Available online at: March 20, 2020

DOI: 10.21608/ijma.2020.23839.1101

\section{ABSTRACT}

Background: Preeclampsia is one of complications of pregnancy. It is responsible for morbidity for mother and fetus. Preeclampsia is a pregnancy multisystem disorder. Abnormal lipid profile has an effect on endothelial dysfunction. The association of serum lipid profile with preeclampsia is suggested to reflect new diagnostic tools. Pregnancy dyslipidemia is associated with an increased risk of preeclampsia. Compared to healthy pregnancy, women with preeclampsia have an abnormal lipid profile.

Aim of the work: The aim of this work is comparison between normotensive women and preeclamptic women by serum lipid profile at third trimester.

Patients and Methods: The present study conducted on 70 pregnant women in the Department of Obstetrics and Gynecology, at Al-Azhar University hospital [Damietta], which was conducted during 2019 [from April to December]. A study comprised 35 normotensive pregnant women as controls and 35 preeclampsia cases. The blood samples were analyzed for serum triglycerides [TGs], total cholesterol [TC], high-density lipoprotein-cholesterol [HDL-C], lowdensity Lipoprotein-Cholesterol [LDL-C], very low-density Lipoprotein-Cholesterol [VLDL-C], Apo lipoprotein- A1 [Apo-A1] and Apo lipoprotein-B[Apo-B].

Results: There was significant difference between group $A$ and $B$ as regard $A p o B / A p o A 1$, cholesterol, TGS, LDL and VLDL. They are higher in group $B$.

Conclusion: In the third trimester of pregnancy, preeclamptic women have altered levels of serum lipid profile. The most significant test is Apo B/A1 ratio with accuracy [72.7\%].

Keywords: Preeclampsia; Lipids; Apolipoprotein; Cholesterol; Triglycerides.

This is an open access article under the Creative Commons license [CC BY] [https://creativecommons.org/licenses/by/2.0/]

Please cite this article as: Mohammed GK, Ayad WA, El-Gazzar UB, Eid SM. Maternal Plasma Lipid Concentration in Third Trimester of Women with Preeclampsia and Normotensive Pregnancy. IJMA 2020; 2[2]: 405-411.

* Main subject and any subcategories have been classified according to researchers' main field of study. 


\section{INTRODUCTION}

Preeclampsia is a pregnancy specific multisystem disorder. It is featured by new onset of hypertension [blood pressure $\geq 140 / 90 \mathrm{mmHg}$ in previously normotensive women after the $20^{\text {th }}$ week of pregnancy] and proteinuria [ $\geq 300 \mathrm{mg} / 24 \mathrm{hr}$ urine collection] $]^{[1]}$. The etiology and development of preeclampsia is abnormal placentation related to immune mechanisms and maladaptation of the placenta. It is clear that one mechanism causing the syndrome preeclampsia does not exist. Instead, many mechanisms can act together and even multiply each other. [2] Abnormal lipid profile is one of causes of atherosclerosis and leads to endothelial abnormality. Hypertension is a mainly significant characteristic in preeclampsia is which cause the vasospasm in the kidney, uterus, placenta, and brain. [3] Dyslipidemia leads to reduction of PGI: TxA2 relation which leads to development of preeclampsia[4] Therefore, dyslipidemia seems significant in the development of preeclampsia. Moreover, the relationship of dyslipidemia with preeclampsia is strongly suggested to reveal recent diagnostic methods. First trimester dyslipidemia is highly related with development of preeclampsia. [5]

Compared to normotensive pregnancy, preeclamptic women have dyslipidemia with high levels of TGs. The possible relation between maternal levels of Apo Band Apo A1and preeclampsia has also been analyzed. Apo lipoproteins are lipoproteins implicated in the provocation of inflammatory process and elimination of overload cholesterol. However, the relation of these metabolites in preeclampsia etiology remains indistinguishable. [6]

\section{AIM OF THE WORK}

In this work we do a comparison between normotensive women and preeclamptic women by serum lipid profile at third trimester. This may help in improvement of fetal and maternal health.

\section{PATIENTS AND METHODS}

Our research included 70 pregnant women from obstetrics and gynecology department [Al-Azhar University hospital, Damietta]. It is a cross-sectional comparative study. Our work was done during 2019 [from April to December]. The study comprised 35 normotensive pregnant women as controls and 35 preeclampsia patients as the study group, from those attending antenatal outpatient department or getting admitted in their last trimester of pregnancy. Informed permissions were taken from whole individuals shared in our research.

\section{Sample size calculation:}

The calculated sample size of the study was determined to be 30 participants for each group at $5 \%$ level of significance and $80 \%$ power, using the following formula. ${ }^{[7]} \mathrm{N}=[\mathrm{Z1}-\alpha / 2+Z 1-\beta]^{2} \sigma 1^{*} \sigma 2 / \delta^{2}$

Where, $\mathrm{Z1}-\alpha / 2=1.96, \mathrm{Z1}-\beta=0.842, \sigma=\mathrm{SD}[0.54,0.29]$, $\delta=$ Expected difference detected among the studied groups regarding LDL/HDL ratio [0.2], $a=$ Level of acceptability of a false positive result [level of significance $=0.05], \beta=$ Level of acceptability of a false negative result [0.20], 1- $\beta=$ power [0.80]. The sample size should be enlarged to 35 pregnant ladies for each group to balance for protocol difficulties with unfinished data and to improve the strength of the research.

The groups of the study were:

Group [A] [controls]: Thirty-five individuals who have the following inclusion criteria: Gravid women, normotensive pregnant woman in the last trimester [gestational age of $>32$ weeks], single live singleton pregnancy and without any other systemic or endocrine disorder, and prepregnancy body mass index $[\mathrm{BMI}] \leq 25$ were included in the study. Group [B] cases: Thirty-five individuals who have the following inclusion criteria: gravid women, preeclamptic pregnant patient in the third trimester [gestational age of $>32$ weeks], single, live singleton pregnancy and pregnant women without any other disease, and prepregnancy body mass index [BMI] $\leq 25$ were integrated in the work.

Exclusion criteria were: diabetics mellitus, obesity, severe anemia [hemoglobin $<6 \mathrm{~g} / \mathrm{dl}$ ], or individuals who have any disease and multiple pregnancy.

Laboratory investigations: we measured the serum levels of the following: [Apo-A1, Apo-B, Apo B/Apo A1 ratio, CHOL, TGS, HDL, LDL and VLDL

\section{Ethical consideration:}

Study protocol was submitted for approval by Institution Research Board [IRB] of the Damietta faculty of Medicine, Al-Azhar University [Egypt]. Informed consent was obtained and confidentiality and personal privacy was respected in all steps of 
the work.

Collection of samples: Seven milliliters of fresh blood were withdrawn from each subject under complete aseptic precautions. $2 \mathrm{ml}$ placed in a tube containing ethylene diamine tetra acetic acid [EDTA] for complete blood count [CBC]. The rest of blood was placed in sterile vacationers and was left to clot for 30 minutes. Serum was then separated by centrifugation at $3000 \mathrm{rpm}$ for 15 minutes and divided into two aliquots; one aliquot for immediate assay of routine lab.

The determination of cholesterol level was carried out as described by Ellefson and Caraway[8]. Determination of Triglycerides level done as described by Roeschlau[9]. Determination of HDL, LDL and VLDL levels done as originally described by Friedewald et al.[10]. Determination of Apo B level was performed as made by Contois et al.[11]. Determinations of Apo A1 level was completed as described by Colantonio et al. [12].

Statistical analysis information was analyzed by the Statistical Package of Social Science [SPSS] program for Windows [Standard version 21]. The normality of information was first analyzed with onesample Kolmogorov-Smirnov test. Qualitative data were presented by using number and percent. Relation among categorical variables was experienced using chi-square test. Permanent variables were described as mean $\pm S D$ [standard deviation] for parametric data. Group $A$ and group $B$ were tested with student t- test. Pearson correlation was made to correlate continuous parametric statistics. Sensitivity and specificity at multiple cut off levels were analyzed by ROC curve. [13]

\section{RESUTLS}

Table [1] shows no significant difference as regard age among two groups [A and $B]$. The mean age for group $A$ and $B$ was [27.83 \pm 5.01 ] and [29.63 \pm 6.81$]$ respectively. There was significant difference as regard $G A$ between group $A$ and $B$ with mean [36.08 \pm 2.27$]$ and [34.48 \pm 2.06$]$ so there is increase of prematurity among preeclamptics [PET]. Table [2] showed significant difference between groups $A$ and $B$ as regard parity and gravidity. PET is significant increase of primigravida [28.6\%] and nullipara [31.4\%] in group B when compared to compared to group A [primigravida [8.6\%] and nullipara [11.4\%]]. On the other side, there was insignificant difference between both groups regarding mode of labor as well as abortion.

Table [3] showed significant difference between groups regarding levels of Apo A1 [the mean values were $94.68 \pm 20.34$ and $77.09 \pm 12.87$ in groups $A$ and $B$ respectively]. However, there was significant decrease of $A p o B / A 1$ ratio in group $A$ when compared to group $B[0.786 \pm 0.22$ vs $1.01 \pm 0.18$ respectively.

Table [4] shows significant difference among two groups in all lipid profile parameters. The level of cholesterol, TGS, LDL and VLDL were higher in group B. However, the level of HDL was low in group $B$ versus group $A$.

Table [5] shows that AUC for Apo A1 was equal to [0.776] with $\mathrm{Cl}$ range from [0.667 to 0.89 .75 ] at cutoff point $[<89.75]$ and sensitivity $[90.9 \%]$ and Specificity [54.3\%]. PPV for Apo A1 was [65.2] and NPV is [86.4] with total accuracy [72.1\%]. In this table, we observed that, AUC for Apo B equal to [0.660] with $\mathrm{Cl}$ range from [0.530 to 0.791$]$ at cutoff point [> 70.5] and sensitivity [67.7\%] and Specificity [51.4\%]. The PPV for Apo B was [57.5] and NPV was [64.3] with total accuracy [60.3\%]. In addition, AUC for Apo B/Apo $\mathrm{A} 1$ ratio was [0.793] with $\mathrm{Cl}$ range from [0.685 to 0.901] at cutoff point [>76.8] and sensitivity [93.5\%] and specificity [54.3\%], PPV was [64.4] and NPV was [90.5] with total accuracy of [72. $7 \%$ ]. The best predictor for PET is Apo B/Apo A1 ratio with accuracy $[72.7 \%]$.

Table [1]: Demographic data among the both groups

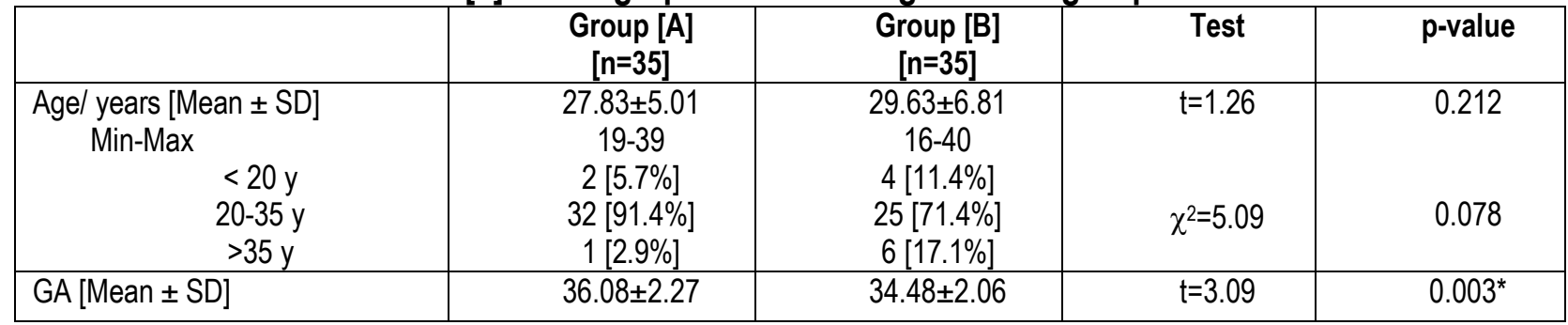

t: student t-test, $\chi^{2}$ : Chi square test; Group [A]: Normotensive group; Group [B]: Preeclampsia group 
Table [2]: Obstetric data among the studied groups

\begin{tabular}{|l|l|c|c|c|c|}
\hline \multicolumn{2}{|c|}{ Obstetric data } & $\begin{array}{c}\text { Group [A] } \\
{[\mathrm{n}=35]}\end{array}$ & $\begin{array}{c}\text { Group [B] } \\
{[\mathrm{n}=35]}\end{array}$ & $\chi^{2}$ & p-value \\
\hline Gravidity & Primigravida & $3[8.6 \%]$ & $10[28.6 \%]$ & 4.63 & $0.031^{*}$ \\
& Multigravida & $32[91.4 \%]$ & $25[71.4 \%]$ & & \\
\hline Parity & Nulipara & $4[11.4 \%]$ & $11[31.4 \%]$ & 4.16 & $0.041^{*}$ \\
& Multipara & $31[88.6 \%]$ & $24[68.6 \%]$ & & \\
\hline Mode of delivery & No & $4[11.4 \%]$ & $11[31.4 \%]$ & 5.47 & 0.065 \\
& CS & $27[77.2 \%]$ & $18[51.4 \%]$ & & \\
& VD & $4[11.4 \%]$ & $6[17.1 \%]$ & & 1.0 \\
\hline Abortion & Yes & $7[20 \%]$ & $7[20 \%]$ & 0 & \\
& No & $28[80 \%]$ & $28[80 \%]$ & & \\
\hline
\end{tabular}

Table [3]: Apo A1, Apo B and Apo B/ A ratio among both studied groups

\begin{tabular}{|l|c|c|c|c|}
\hline Variables & $\begin{array}{c}\text { Group [A] } \\
{[\mathrm{n}=35]}\end{array}$ & $\begin{array}{c}\text { Group [B] } \\
{[\mathrm{n}=35]}\end{array}$ & t-test & p-value \\
\hline Apo A1 & $94.68 \pm 20.34$ & $77.09 \pm 12.87$ & 4.23 & $<0.001^{*}$ \\
\hline Apo B & $71.77 \pm 18.01$ & $79.14 \pm 14.18$ & 1.86 & 0.066 \\
\hline Apo B/ A ratio & $0.786 \pm 0.22$ & $1.01 \pm 0.18$ & 4.28 & $<0.001^{*}$ \\
\hline
\end{tabular}

Table [4]: Lipid profile among the studied groups

\begin{tabular}{|c|c|c|c|c|}
\hline Lipid profile & $\begin{array}{c}\text { Group [A] } \\
{[\mathrm{n}=35]}\end{array}$ & $\begin{array}{c}\text { Group [B] } \\
{[\mathrm{n}=35]}\end{array}$ & Test of significance & $\mathbf{p}$-value \\
\hline Cholesterol & $135.82 \pm 6.45$ & $153.73 \pm 13.37$ & 7.138 & $<0.001^{*}$ \\
\hline TGS & $122.59 \pm 20.23$ & $141.42 \pm 45.71$ & 2.229 & $0.029^{*}$ \\
\hline HDL & $43.14 \pm 3.19$ & $23.23 \pm 4.46$ & 21.491 & $<0.001^{*}$ \\
\hline LDL & $68.35 \pm 15.41$ & $102.22 \pm 14.21$ & 9.558 & $<0.001^{*}$ \\
\hline VLDL & $24.52 \pm 5.13$ & $28.28 \pm 7.36$ & -2.485 & $0.015^{*}$ \\
\hline
\end{tabular}

Table [5]: Diagnostic accuracy of Apo A1, Apo B and Apo B/ A ratio in prediction of preeclampsia

\begin{tabular}{|l|c|c|c|c|c|c|c|c|c|}
\hline & AUC & \multicolumn{2}{|c|}{$95 \%$ Cl } & Cutoff & Sensitivity & Specificity & PPV & NPV & Accuracy \\
\cline { 3 - 8 } & & Lower & Upper & & & & & & \\
\hline Apo A1 & 0.776 & 0.667 & 0.885 & $<89.75$ & $90.9 \%$ & $54.3 \%$ & 65.2 & 86.4 & $72.1 \%$ \\
\hline Apo B & 0.660 & 0.530 & 0.791 & $>70.5$ & $67.7 \%$ & $51.4 \%$ & 57.5 & 64.3 & $60.3 \%$ \\
\hline Apo B/ A1 ratio & 0.793 & 0.685 & 0.901 & $>76.8$ & $93.5 \%$ & $54.3 \%$ & 64.4 & 90.5 & $72.7 \%$ \\
\hline
\end{tabular}

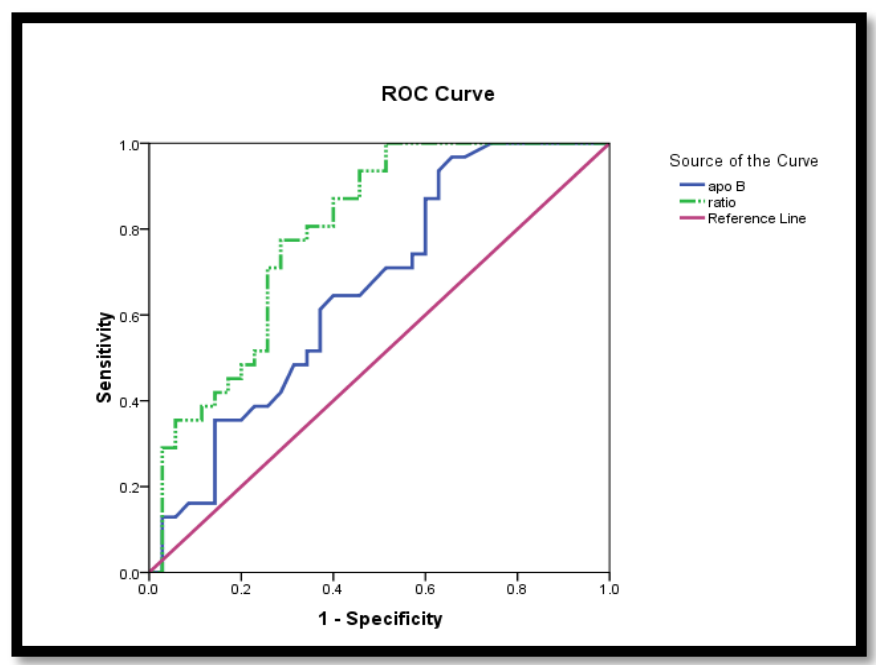

Figure [1]: Receiver operating characteristics curve [ROC] for Apo B and Apo B/ A ratio in prediction of preeclampsia

\section{DISUCSSION}

Our work was carried out to compare between normotensive women and preeclamptic women by serum lipid profile at third trimester. This may help in improvement of fetal and maternal health.
From our results, we could say that pregnant women with abnormal lipid profile [dyslipidemia] are more prone for preeclampsia. Lipids significantly differ between groups. Comparable results were 
discovered by Nagtilak. High lipid profile levels lead to injury of the endothelium. In the endothelium, oxidative stress is motivated by linoleic acid. Maternal lipoprotein levels rise considerably and are twice higher in PET. In addition, physiological changes that occur during pregnancy consist of insulin resistance, hyperlipidemia, and up regulation of inflammatory markers. Both mechanisms could share in elevated blood pressure. [13]

In a research by Gratacos et al. [14], cholesterol levels were not significantly elevated in preeclamptic patients, but triglyceride levels were significantly elevated from two months. This difference could be attributed to different inclusion criteria and variable sample size, and timing of determination of lipid profile.

Adiga et al.[15] observed that serum TGs was statistically significant in preeclamptic women in comparison with normal pregnancy. The main modulator of this high TGs is high estrogen level in pregnancy. Elevated TGs levels may be associated with hypercoagulability.

In many works, significantly elevated levels of TGs in PET were detected in comparison with healthy pregnant women were concluded which is in high harmony with larger studies. [13, 15-18]

Enquobahrie et al.[5] measured serum lipid level in 57 patients with preeclampsia and 510 pregnant women in the control group and they discovered significantly raised levels of TGs in preeclampsia. [5] In another research, preeclamptic women had important high levels of TGs than in healthy group. Mikhail et al. concluded that elevated TGs in serum causes its major deposition in the endothelium. This may lead to unhealthy endothelium in pregnant women. [19]

In another study done in Spain, at the second trimester, triglyceride levels were significantly elevated in severe PET than in controls. This is also in harmony with the present study. [13]

Increased TGs in PET are liable to be deposited in uterine spiral arteries and cause injury of the endothelium, by direct mechanism and indirect mechanism throughout production of small, dense LDL. [14]

In a study of Wakatsuki et al.[20], HDL-C levels had no significant difference among women with
PET and healthy pregnant women. This could be attributed to different sample size and time of sampling for lipid profile measurement.

LDL-C was higher among cases when compared to controls [102.22 \pm 14.21 vs $68.35 \pm 15.41$ respectively], the results were previously reported by Mishra et al.[21] Kandimalla et al.[22] concluded that LDL-C measurements were established to be significantly elevated in the women with PET.

In the current study, VLDL measurements were significantly $[P<0.088]$ high in the preeclamptic group which may be as a result of high TGs levels causing high entrance of VLDL that carries the endogenous TGs into the circulation. Similar results were seen in the research done via Vani et al.[23]

In contradiction to results of the present work, Ghodke et al.[24] are not capable to expect the incidence of PET, diabetes, and PRL by measuring VLDL-C levels of the mid trimester as the level are within normal concentration.

The Apo $E$ isoforms and gene variants have been postulated and used as probable predictors of PET development. However, the detailed effect of these metabolites in preeclampsia etiology residue uncertain[25]. Furthermore, preeclampsia has a positive correlation with $\mathrm{Apo} B / \mathrm{ApoA} 1$ ratio, and negative correlation with ApoA1. [26]

Current work revealed elevated Apo B/A1 ratio and Apo $B$ levels among women with PET in comparison with normotensive women but Apo A1 levels were lower in preeclamptic group. Charlton et al. ${ }^{27]}$ work explored the possibility of Apo A1, a main lipoprotein component of $\mathrm{HDL}$, to improve or reverse the hypertension and placental changes discovered in an animal model of inflammatory cytokine-induced hypertension in pregnancy. They further examined whether Apo A1 has a protective effect in an in vitro model of human being trophoblast invasion and whether this is owing to alterations in adhesion molecules and markers of invasion. The protective effect conferred by Apo A1 on the cytokine-induced hypertension in pregnancy is suggested to be owing to tissue uptake rather than its level in the blood. [27]

Apo A1 mimetic peptides show anti-inflammatory and antioxidant criteria and function by stimulating cholesterol efflux and reverse cholesterol transport via ABCA1. [28,29] Inflammation and oxidative stress are key potential mechanisms that could make good 
changes in the stiffness of arteries. [30,31] Therefore, anti-inflammatory drugs are recommended to recover arterial stiffness as a potential therapy, while favorable outcome have been revealed in several studies. [32-34]

\section{Conclusion:}

In the third trimester of pregnancy, preeclamptic women have altered levels of serum lipid profile when compared to females with normal blood pressure. The highest significant test is Apo B/A1 ratio with accuracy $[72.7 \%]$.

Financial and Non-Financial Relationships and Activities of Interest

None

\section{REFERENCES}

1. Fox R, Kitt J, Leeson P, Aye CYL, Lewandowski AJ. Preeclampsia: Risk Factors, Diagnosis, Management, and the Cardiovascular Impact on the Offspring. J Clin Med. 2019 Oct 4;8[10]. pii: E1625. [DOI: 10.3390/jcm8101625].

2. Shamsi U, Saleem S, Nisther N. Epidemiology and risk factors of preeclampsia; an overview of observational studies. Al-Ameen J Med Sci. 2013; 6 [4]: 292-300.

3. Lindheimer MD, cunningham FG, Roberts JM. Introduction, History, controversies, and Definitions in Chelsey's Hypertensive Disorders in Pregnancy. Amsterdam, Netherlands: Elsevier; 2009; pp. 1-24.

4. Simmons LA, Gillin AG, Jeremy RW. Structural and functional changes in left ventricle during normotensive and preeclamptic pregnancy. Am J Physiol Heart Circ Physiol; 2002;283:H1627-33. [DOI: 10.1152/ajpheart.00966.2001].

5. Enquobahrie DA, Williams MA, Butler $\mathrm{Cl}$, Frederick 10, Miller RS, Luthy DA. Maternal plasma lipid concentrations in early pregnancy and risk of preeclampsia. Am J Hyperten. 2004;17:574-581. [DOI: 10.1016/j.amjhyper.2004.03.666].

6. Gallos I, Sivakumar K, Kilby M, Coomarasamy A, Thangaratinam S, Vatish M. Preeclampsia is associated with, and preceded by, hypertriglyceridaemia: a meta-analysis. BJOG 2013;120:1321-1332. [DOI:10.1111/1471-0528.12375].

7. Kirby A, Gebski V, Keech AC. Determining the sample size in a clinical trial. Med J Aust. 2002; 177:256-7. [PMID:12197821].

8. Ellefson RD, Caraway WT. Fundamentals of clinical chemistry EdTietz NW 1976; p506.

9. Roeschlau P, Bernt E, Gruber WJ. Enzymatic determination of total cholesterol in serum. Clin Chem
Clin Brioche.1974; 12:403. [PMID:4440114].

10. Friedewald WT, Levy RI, Fredrickson DS. Estimation of the concentration of low-density lipoprotein cholesterol in plasma, without use of the preparative ultracentrifuge. Clin Chem. 1972, 18;499502. [PMID: 4337382].

11. Contois JH, Mcconnell JP, Sethi AA, Csako G, Devaraj S, Hoefner DM, et al. Apolipoprotein B and cardiovascular disease risk: Position statement from the AAcc Lipoproteins and Vascular Diseases Division Working Group on Best Practices. Clin Chem. 2009; 55[3]:407-419. [DOI: 10.1373/clinchem. 2008. 118356].

12. Colantonio DA, Kyriakopoulou L, Chan MK, Daly $\mathrm{CH}$, Brinc D, Venner AA, et al. Closing the gaps in pediatric laboratory reference intervals: A caliper database of 40 biochemical markers in a healthy and multiethnic population of children. Clin Chem. 2012; 58[5]:854-868. [DOI: 10.1373/dlinchem.2011. 177741].

13. Nagtilak S. The levels of serum lipid profile and free fatty acids in normotensive and preeclamptic pregnant women in their third trimester pregnancy. Int J Basic Med Clin Res. 2015; 2: 65-134

14. Gratacos E, casals E, Sanllehy c, cararach V, Alonso PL, Fortuny A. Variation in lipid levels during pregnancy in women with different types of hypertension. Acta obstetricia et gynecologica Scandinavica 1996; 75:896-901. [DOI:10.3109/000 16349609055024].

15. Adiga U, Dsouza V, Kamath A, Mangalore N. Antioxidant Activity and Lipid Peroxidation in Preeclampsia. J Chin Med Assoc. 2007; 70[10]: 435438. [DOI: 10.1016/S1726-4901[08]70034-0].

16. Lorentzen B, Drevon CA, Endresen MJ, Henriksen $\mathbf{T}$. Fatty acid pattern of esterified and free fatty acids in sera of women with normal and pre-eclamptic pregnancy. BJOG. 1995;102: 530-537. [DOI: 10.1111/ j.1471- 0528. 1995.tb11355.x].

17. Sattar N, Bendomir A, Berry C, Shepherd J, Greer IA, Packard CJ. Lipoprotein sub-fraction concentrations in preeclampsia: pathogenic parallels to atherosclerosis. Obstetr Gynecol.1997; 89:403408. [DOI: 10.1016/S0029-7844[96]00514-5].

18. Ziaei S, Motlagh Bonab K, Kazemnejad A . Serum lipid levels at 28-32 weeks gestation and hypertensive disorders. Hypertens Pregnancy 2006; 25:3-10. [DOI: 10.1080/10641950500543756].

19. Mikhail M, Basu J, Palan P, Furgiuele J, Romney S, Anyaegbunam A. Lipid profile in women with preeclampsia: relationship between plasma triglyceride levels and severity of preeclampsia. J Assr Acad Minor Phys. 1995; 6:43-45. [PMID:7858376].

20. Wakatsuki A, Ikenoue N, Okatani Y, Shinohara K, Fukaya T. Lipoprotein particles in preeclampsia: 
susceptibility to oxidative modification. Obstetr Gynecol 2000; 96 [1]:55-59. [DOI:10.1016/s00297844[00]00858-9].

21. Mishra MK, Sharma R, Sharma D, Mishra A, Sharma D. Study of variations in lipid profile in different trimesters of pregnancy. Int J Med Sci Public Health 2017; 6:456-460. [DOI: 10.5455/ijmsph.2017. 0525911052016].

22. Kandimalla BH, Sirjusingh A, Nayak BS, Maiya SS. Early antenatal serum lipid levels and the risk of preeclampsia in Trinidad and Tobago. Arch Physiol Biochem $2011 ; 117: 215-221$. [DOI; 10.3109/ 13813455. 2010.543137]

23. Vani I, Gayathri A, NagamaniT, Sunieeta H. Lipid profile parameters in normal and preeclampsia complicating pregnancies: A prospective study. Am J Sci Med Res 2015; 1:61-6. [DOI:10.17812/ajsmr 2015114].

24. Ghodke B, Pusukuru R, Mehta V. Association of Lipid Profile in Pregnancy with Preeclampsia, Gestational Diabetes Mellitus, and Preterm Delivery. Cureus. 2017; 9: e1420. [DOI:10.7759/cureus.1420].

25. Carty DM, Delles C, Dominiczak AF. Novel biomarkers for predicting preeclampsia. Trends Cardiovasc Med. 2008; 18:186-194. [DOI: 10.1016/ j.tcm. 2008.07.002].

26. Serrano NC, Guio-Mahecha E, Quintero-Lesmes DC, Becerra-Bayona S, Paez MC, Beltran M, et al. Lipid profile, plasma apolipoproteins, and preeclampsia risk in the GenPE. case-control study. Atherosclerosis 2018; 276:189-194. [DOI; 10.1016/ j. atherosclerosis.2018.05.051].

27. Charlton F, Bobek G, Stait-Gardner T, Price WS, Colafella KM, et al. The protective effect of apolipoprotein in models of trophoblast invasion and preeclampsia. Am J Physiol-Regul Integr Comp Physiol 2017 ;312:R40-R48. [ DOI: 10.1152/ajpregu. 00331.2016].
28. Getz GS, Reardon cA. The structure/function of apoprotein Al mimetic peptides: an update. Curr Opin Endocrinol Diabetes Obes. 2014 Apr; 21[2]:129-33. [DOI: 10.1097/MED.0000000000000045[.

29. Tabet F, Remaley AT, Segaliny Al, Millet J, Yan L, Nakhla S, et al. The $5 \mathrm{~A}$ apolipoprotein Al mimetic peptide displays antiinflammatory and antioxidant properties in vivo and in vitro. Arterioscler Thromb Vasc Biol. 2010; 30:246-252. [DOI:10.1161/ ATVBAHA.109.200196].

30. Jain S, Khera R, Corrales-Medina VF, Townsend RR, Chirinos JA. Inflammation and arterial stiffness in humans. Atherosclerosis 2014; 237:381-390. [DOI: 10.1016/j.atherosclerosis.2014.09.011]

31. Patel RS, Al Mheid I, Morris AA, Ahmed Y, Kavtaradze N, Ali $\mathbf{S}$, et al. Oxidative stress is associated with impaired arterial elasticity. Atherosclerosis 2011; 218:90-95. [doi: 10.1016/ j. atherosclerosis.2011.04.033]

32. Ichihara A, Hayashi M, Koura Y, Tada Y, Kaneshiro Y, Saruta T. Long-term effects of statins on arterial pressure and stiffness of hypertensives. J Human Hyperten 2005; 19:103. [DOI:10.1038/sj. jhh. 1001786].

33. Matsuo T, Iwade K, Hirata N, Yamashita M, Ikegami H, Tanaka N, Aosaki M, Kasanuki H. Improvement of arterial stiffness by the antioxidant and antiinflammatory effects of short-term statin therapy in patients with hypercholesterolemia. Heart Vessels 2005; 20:8-12. [DOI 10.1007/s00380-004-0793-5].

34. Meng X, Qie L, Wang Y, Zhong M, Li L [2009] Assessment of arterial stiffness affected by atorvastatin in coronary artery disease using pulse wave velocity. Clin Invest Med. 2009; E238-E243 